\title{
Effect of bulk Lorentz violation on anisotropic brane cosmologies
}

\author{
Malihe Heydari-Fard*† \\ Department of Physics, The University of Qom, P. O. Box 37155-1814, Qom, Iran
}

August 8, 2018

\begin{abstract}
The effect of Lorentz invariance violation in cosmology has attracted a considerable amount of attention. By using a dynamical vector field assumed to point in the bulk direction, with Lorentz invariance holding on the brane, we extend the notation of Lorentz violation in four dimensions $[28]$ to a five-dimensional brane-world. We obtain the general solution of the field equations in an exact parametric form for Bianchi type I space-time, with perfect fluid as a matter source. We show that the brane universe evolves from an isotropic/anisotropic state to an isotropic de Sitter inflationary phase at late time. The early time behavior of anisotropic brane universe is largely dependent on the Lorentz violating parameters $\beta_{i}, i=1,2,3$ and the equation of state of the matter, while its late time behavior is independent of these parameters.
\end{abstract}

PACS numbers: 98.80.-k, 11.30.Cp, 04.50.-h

Key words: Anisotropy cosmologies, Lorentz invariance violation, Brane-world

\section{Introduction}

Brane-world scenarios with a 3-brane identified with the observable universe which is embedded in a higher-dimensional space-time provide an alternative to the standard cosmology in four-dimension $[1,2,3]$. A essential requirement on these models is that they should reproduce the important observational cosmological data, the age of the universe, abundances of elements produced in primordial nucleosynthesis, etc. One of the most successful of such higher-dimensional models is that proposed by Randall and Sundrum whose the bulk has the geometry of an AdS space admitting $Z_{2}$ symmetry [1]. They were successful in explaining the hierarchy problem: the enormous disparity between the strength of the fundamental forces. The Randall Sundrum (RS) model has had a large impact on our undersetting of the universe and has brought higher-dimensional gravitational theories to the fore. In RS type models, all matter and gauge fields live on the brane, while gravity can propagate into the bulk. Using the Israel junction conditions [4] and the Gauss-Codazzi equations, one can obtain the gravitational field equations on the brane, as employed by Shiromizu, Maeda and Sasaki (SMS) [5]. There are two important difference that result from the effective four-dimensional gravitational equations on the brane. The first one is quadratic energy-momentum tensor, $\pi_{\mu \nu}$, which is important in a high-energy universe and the second one is the projected Weyl tensor, $\mathcal{E}_{\mu \nu}$, on the brane which is responsible for carrying on the brane the contribution of the bulk gravitational field. The cosmological evolution of such a brane universe has been investigated and effects such as a quadratic density term in the Friedmann equations have been found $[6,7,8]$.

Lorentz symmetry is assumed to be the exact symmetry of nature [9]. However, many exotic theories such as canonical quantum gravity and string theory suggest that Lorentz invariance may

*E-mail: heydarifard@qom.ac.ir

${ }^{\dagger}$ E-mail: m.heydarifard@mail.sbu.ac.ir 
be broken at high energies $[10,11]$. String theory predicts that we may live in a universe with non-commutative coordinate [12] leading to violation of Lorentz invariance as an explanation for the astrophysical anomalies such as the missing Greisen-Zatsepin-Kuzmin cutoff $[13,14,15,16]$. A method of implementing the local Lorentz violation in a gravitational setting is to consider the existence of a tensor field with a non-vanishing expectation value and then couple it to gravity or matter fields. The simplest example of this method is to consider a single time-like vector field with fixed norm. A special case of this theory as a mechanism for Lorentz invariance violation has been introduced by Kostelecky and Samuel [17]. In a different context, a theory of gravity with a fixed norm vector in order to mimic the effects of dark matter has been proposed by Bekenstein [18]. Also, for the studies of vector fields in a cosmological setting without the fixed norm see $[19,20]$. This vector field picks out a preferred frame at each point in space-time and any matter field coupled to this vector field will experience a violation of local Lorentz invariance [21]. However, in curved space-time there is no natural generalization of the notion of a constant vector field $\left(\nabla_{\mu} N^{\nu}=0\right.$ generically has no solutions); therefore we must allow that the vector field to have dynamic and fix the norm of it by choosing an appropriate action for the field.

The effects of Lorentz violation have been also studied within the context of the brane-world scenarios [22]. In these models, the space-time violates four-dimensional Lorentz invariance globally and leads to apparent violations of Lorentz invariance from the brane observers point of view due to bulk gravity effects. These effects are restricted to the gravity sector of the effective theory while the measured Lorentz invariance of particle physics remains unaffected in these models [23]. In a similar way, Lorentz invariance violation has had to shed some light on the possibility of signals travelling along the extra dimension outside our universe [24]. In a different method a brane-world toy model in an inflating five-dimensional brane-world scenario has been introduced with violation of four-dimensional Lorentz invariance at an energy scale $k$ [25]. In [26], the authors have studied Lorentz violation in a brane-world model by considering a vector field normal to the brane along the extra dimension. They showed that local Lorentz violation in the bulk allows for the construction of models in which the vacuum energy, the gravitational coupling and the cosmological term on the brane are variable. There also exist a relation between the maximal velocity in the bulk and the speed of light on the brane [27]. The main aim of this paper is to see how the shear parameter behaves in such brane-world scenario and whether this model isotropises at late time. To achieve this goal we study the local Lorentz invariance violation in the same setting and investigate the behavior of the observationally important parameters like shear, anisotropy, and the deceleration parameter in a Bianchi type I geometry.

\section{The setup}

Let us start by presenting the model used in our calculations. We only state the results and refer the reader to [26] for a detailed derivation of these results.

As mentioned above, the brane-world model we consider here includes Lorentz violation in the bulk space along the extra dimension by generalizing the theory suggested by Jacobson and Mattingly $[28,29]$, where the quartic self-interaction term $\left(N^{A} \nabla_{A} N^{B}\right)\left(N^{C} \nabla_{C} N_{B}\right)$ has been ignored [30]. We assume that $N^{A}$ is a vector field along the extra dimension which is making the associated frame a preferred one. We also consider that the theory which is consists of the vector field $N^{A}$ minimally coupled to gravity with the following action

$$
\mathcal{S}=\int d^{5} x \sqrt{-g^{(5)}}\left[\frac{1}{2 \kappa_{5}^{2}}\left(R^{(5)}+\mathcal{L}_{N}\right)+\mathcal{L}_{m}\right]
$$

where $\kappa_{5}^{2}=8 \pi G_{5}, R^{(5)}$ is the five-dimensional Ricci scalar, $\mathcal{L}_{N}$ is the vector field Lagrangian density and $\mathcal{L}_{m}$ is the Lagrangian density for the matter fields. We take $\mathcal{N}^{A}$ as a dynamical field for preserving the general covariance. The Lagrangian density for the vector field is given by

$$
\mathcal{L}_{N}=K_{C D}^{A B} \nabla_{A} N^{C} \nabla_{B} N^{D}+\lambda\left(N_{A} N^{A}-\epsilon\right),
$$


where $\lambda$ is a Lagrange multiplier and

$$
K_{C D}^{A B}=-\beta_{1} g^{A B} g_{C D}-\beta_{2} \delta_{C}^{A} \delta_{D}^{B}-\beta_{3} \delta_{D}^{A} \delta_{C}^{B},
$$

here, $\beta_{i}, i=1,2,3$, dimensionless parameters, $\epsilon=-1$ or $\epsilon=+1$ depending on whether the extra dimension is time-like or space-like, respectively. Using the metric of the bulk space as

$$
d S^{2}=g_{\mu \nu}\left(x^{\alpha}, y\right) d x^{\mu} d x^{\nu}+\epsilon \phi\left(x^{\alpha}, y\right) d y^{2},
$$

and

$$
g_{A B} N^{A} N^{B}=\epsilon, \quad \epsilon^{2}=1,
$$

and assuming that the normal unit vector $N^{A}$ which is orthogonal to the hypersurfaces $y=$ const. as, $N^{A}=\frac{\delta_{5}^{A}}{\phi}$, the gravitational field equations on the brane become as $[26]$

$$
\begin{aligned}
G_{\mu \nu} & =\frac{k_{5}^{2}}{2} g_{\mu \nu} \Lambda_{5}-\frac{3\left(\epsilon+\alpha_{1}\right)}{\left(3+\alpha_{1}\right)}\left(K_{\mu \gamma} K_{\nu}^{\gamma}-K K_{\mu \nu}\right)-\frac{3\left(\epsilon+\alpha_{3}\right)}{2\left(3+\alpha_{1}\right)} g_{\mu \nu} K^{2} \\
& +\frac{3\left(\epsilon+\alpha_{4}\right)}{2\left(3+\alpha_{1}\right)} g_{\mu \nu} K_{\alpha \beta} K^{\alpha \beta}+\left[\frac{\alpha_{1}\left(\alpha_{5}+\frac{1}{2}\right)+3 \alpha_{5}}{\left(3+\alpha_{1}\right)}\right] g_{\mu \nu} \frac{\phi_{; \alpha}^{\alpha}}{\phi}-\frac{\alpha_{5}\left(5+\alpha_{1}\right)}{2\left(3+\alpha_{1}\right)} g_{\mu \nu} \frac{\phi_{, \alpha} \phi_{,}^{\alpha}}{\phi^{2}} \\
& -\frac{2 \alpha_{1}}{\left(3+\alpha_{1}\right)} \frac{\phi_{; \nu \mu}}{\phi}+\frac{4 \alpha_{5}}{\left(3+\alpha_{1}\right)} \frac{\phi_{, \mu} \phi_{, \nu}}{\phi^{2}}-\frac{3\left(\epsilon+\alpha_{1}\right)}{\left(3+\alpha_{1}\right)} \mathcal{E}_{\mu \nu},
\end{aligned}
$$

where

$$
\begin{aligned}
& \alpha_{1}=2\left(\beta_{1}+\beta_{3}\right), \quad \alpha_{2}=\frac{2 \epsilon\left(\beta_{1}+\beta_{2}+\beta_{3}\right)}{3-2 \epsilon\left(\beta_{1}+4 \beta_{2}+\beta_{3}\right)}, \\
& \alpha_{3}=\frac{\alpha_{1}\left(3+\epsilon-2 \beta_{2}\right)}{6}-\beta_{2}, \quad \alpha_{4}=\frac{\alpha_{1}\left(6+\epsilon+\alpha_{1}\right)}{6}, \quad \alpha_{5}=\epsilon \beta_{1} .
\end{aligned}
$$

The first partial derivatives in terms of the extrinsic curvature of the brane is given by

$$
K_{\mu \nu}=\frac{1}{2} \mathcal{L}_{N} g_{\mu \nu}=\frac{1}{2 \phi} \frac{\partial g_{\mu \nu}}{\partial y}, \quad K_{A 5}=0
$$

and

$$
\mathcal{E}_{\mu \nu}=C_{\mu A \nu B}^{(5)} N^{A} N^{B} .
$$

Using equation (6) and the $Z_{2}$ symmetry, we obtain the gravitational field equations on the brane as

$$
\begin{aligned}
G_{\mu \nu} & =-\Lambda_{4} g_{\mu \nu}+\kappa_{4}^{2} \tau_{\mu \nu}+\kappa_{5}^{4} \pi_{\mu \nu}-\frac{3\left(\epsilon+\alpha_{1}\right)}{\left(3+\alpha_{1}\right)} \mathcal{E}_{\mu \nu}+F_{\mu \nu}+\left[\frac{\alpha_{1}\left(\alpha_{5}+\frac{1}{2}\right)+3 \alpha_{5}}{\left(3+\alpha_{1}\right)}\right] g_{\mu \nu} \frac{\phi_{; \alpha}^{\alpha}}{\phi} \\
& -\frac{\alpha_{5}\left(5+\alpha_{1}\right)}{2\left(3+\alpha_{1}\right)} g_{\mu \nu} \frac{\phi_{, \alpha} \phi_{,}^{\alpha}}{\phi^{2}}-\frac{2 \alpha_{1}}{\left(3+\alpha_{1}\right)} \frac{\phi_{; \nu \mu}}{\phi}+\frac{4 \alpha_{5}}{\left(3+\alpha_{1}\right)} \frac{\phi_{, \mu} \phi_{, \nu}}{\phi^{2}}
\end{aligned}
$$

where

$$
\begin{gathered}
\Lambda_{4}=\frac{\kappa_{5}^{2}}{2} \Lambda_{5}+\kappa_{5}^{4}\left[\frac{\epsilon-3\left(-\alpha_{1}+4 \alpha_{6}+16 \alpha_{7}+2 \alpha_{4}\right)}{4\left(3+\alpha_{1}\right)\left(1+\epsilon \alpha_{1}\right)^{2}}\right] \lambda^{2} \\
\kappa_{4}^{2}=\kappa_{5}^{4}\left[\frac{2 \epsilon-3\left(-2 \alpha_{1}+4 \alpha_{6}\right)}{4\left(3+\alpha_{1}\right)\left(1+\epsilon \alpha_{1}\right)^{2}}\right] \lambda \\
\pi_{\mu \nu}=\frac{3}{4\left(3+\alpha_{1}\right)\left(1+\epsilon \alpha_{1}\right)^{2}}\left[-\left(\epsilon+\alpha_{1}\right) \tau_{\mu \alpha} \tau_{\nu}^{\alpha}+\left(\frac{\epsilon}{3}+\alpha_{6}\right) \tau \tau_{\mu \nu}-\left(\frac{\epsilon}{6}-\alpha_{7}\right) g_{\mu \nu} \tau^{2}+\frac{\left(\epsilon+\alpha_{4}\right)}{2} g_{\mu \nu} \tau_{\alpha \beta} \tau^{\alpha \beta}\right] \\
+\left[\frac{3\left(\alpha_{6}+8 \alpha_{7}+\alpha_{4}\right)}{4\left(3+\alpha_{1}\right)\left(1+\epsilon \alpha_{1}\right)^{2}}\right] g_{\mu \nu} \lambda \tau
\end{gathered}
$$




$$
F_{\mu \nu}=\left.\left[\frac{\kappa_{5}^{2}}{2} g_{\mu \nu} \mathcal{T}_{y}^{y}+\frac{2 \kappa_{5}^{2}}{3}\left(\mathcal{T}_{\mu \nu}-\frac{1}{4} g_{\mu \nu} \mathcal{T}_{\alpha}^{\alpha}\right)\right]\right|_{y=0},
$$

and

$$
\begin{gathered}
\alpha_{6}=\frac{\alpha_{1}\left(1-2 \alpha_{2}\right)-2 \epsilon \alpha_{2}}{3}, \\
\alpha_{7}=\frac{\left(\epsilon+\alpha_{1}\right)\left(\alpha_{2}+\alpha_{2}^{2}\right)}{3}+\frac{\left(\alpha_{4}-3 \epsilon-4 \alpha_{3}\right)\left(\alpha_{2}+2 \alpha_{2}^{2}\right)}{9}-\frac{\left(\alpha_{3}+2 \alpha_{4}\right)}{18} .
\end{gathered}
$$

For latter convenience we chose $\alpha_{0}=2 \beta_{2}$, so that constants $\alpha_{i}(i=2, \ldots, 7)$ are defined as a function of $\alpha_{0}$ and $\alpha_{1}$. Now, by considering $N_{5}^{A}=\delta_{5}^{A}, \epsilon=+1$ and ignoring the bulk matter, $F_{\mu \nu}=0$, we obtain the gravitational field equations in four-dimensions as

$$
G_{\mu \nu}=-\Lambda_{4} g_{\mu \nu}+\kappa_{4}^{2}\left(\tau_{\mu \nu}+\frac{\alpha_{1}}{12} g_{\mu \nu} \tau\right)+\kappa_{5}^{4} \pi_{\mu \nu}-\tilde{\mathcal{E}}_{\mu \nu}
$$

where

$$
\begin{gathered}
\Lambda_{4}=\frac{\kappa_{5}^{2}}{2} \Lambda_{5}+\frac{\kappa_{5}^{4}}{4\left(3-4 \alpha_{0}-\alpha_{1}\right)} \lambda^{2} \\
\kappa_{4}^{2}=\frac{3 \kappa_{5}^{4}}{2\left(3+\alpha_{1}\right)\left(3-4 \alpha_{0}-\alpha_{1}\right)} \lambda \\
\pi_{\mu \nu}=\frac{3}{4\left(3+\alpha_{1}\right)\left(1+\alpha_{1}\right)}\left[\frac{\left(1-2 \alpha_{0}-\alpha_{1}\right)}{\left(3-4 \alpha_{0}-\alpha_{1}\right)} \tau \tau_{\mu \nu}-\tau^{\alpha}{ }_{\mu} \tau_{\alpha \nu}+\frac{\left(6+\alpha_{1}\right)}{12} g_{\mu \nu} \tau_{\alpha \beta} \tau^{\alpha \beta}\right. \\
\left.-\frac{2\left(3-\alpha_{1}\right)-\left(9+\alpha_{1}\right) \alpha_{0}}{12\left(3-4 \alpha_{0}-\alpha_{1}\right)} g_{\mu \nu} \tau^{2}\right],
\end{gathered}
$$

and

$$
\tilde{\mathcal{E}}_{\mu \nu}=\frac{3\left(1+\alpha_{1}\right)}{\left(3+\alpha_{1}\right)} \mathcal{E}_{\mu \nu}
$$

We note that the model is different from the SMS model in two cases. The first one is the existence of the trace part of the brane energy-momentum tensor in the modified gravitational field equations on the brane. This trace part of the energy-momentum tensor vanishes when $\alpha_{1}=0$. The second departure from the SMS model arises from definition of the fundamental quantities $\Lambda_{4}$ and $\kappa_{4}^{2}$ which contain higher-dimensional modifications to the standard general relativity.

The Codazzi equation also implies the conservation of the energy-momentum tensor of the matter on the brane

$$
\nabla_{\mu} \tau_{\nu}^{\mu}+\alpha_{2} \nabla_{\nu} \tau-\left(1+4 \alpha_{2}\right) \nabla_{\nu} \lambda=-2\left(\epsilon+\alpha_{1}\right) \mathcal{T}_{\nu}^{y},
$$

thus the brane energy-momentum tensor $\tau_{\nu}^{\mu}$ is not conserved. Moreover, the contracted Bianchi identities on the brane imply that the projected Weyl tensor should obey the constraint

$$
\nabla_{\mu} \tilde{\mathcal{E}}^{\mu}{ }_{\nu}=-\nabla_{\nu} \Lambda_{4}+\kappa_{4}^{2}\left(\nabla_{\mu} \tau^{\mu}{ }_{\nu}+\frac{\alpha_{1}}{12} \nabla_{\nu} \tau\right)+\kappa_{5}^{4} \nabla_{\mu} \pi^{\mu}{ }_{\nu}+\frac{\kappa_{5}^{2}}{2} \nabla_{\nu} \mathcal{T}_{y}^{y}+\frac{2 \kappa_{5}^{2}}{3}\left(\nabla_{\mu} \mathcal{T}_{\nu}^{\mu}-\frac{1}{4} \nabla_{\nu} \mathcal{T}^{\alpha}{ }_{\alpha}\right)
$$

For convenience we suppose that the bulk cosmological constant and brane tension is constant. Thus equations (22) and (23) become

$$
\begin{gathered}
\nabla_{\mu} \tau_{\nu}^{\mu}=-\alpha_{2} \nabla_{\nu} \tau \\
\nabla_{\mu} \tilde{\mathcal{E}}_{\nu}^{\mu}=-\kappa_{4}^{2}\left(\alpha_{2}-\frac{\alpha_{1}}{12}\right) \nabla_{\nu} \tau+\kappa_{5}^{4} \nabla_{\mu} \pi_{\nu}^{\mu} .
\end{gathered}
$$

Equations (17), (24) and equation (25) give a complete set of field equations for the brane gravitational field. In the next section, we study the cosmological consequence of anisotropic brane in the framework of such model. 


\section{Bianchi type I brane cosmology}

In the following we will investigate the influence of the Lorentz violating parameters on the anisotropic universe described by Bianchi type I geometry. Considering $\beta_{i}=0, i=1,2,3$, we can reduce the model to the SMS brane-world model and compare our results to this model.

The line-element of a Bianchi type I space-time, which is a generalization of the isotropic flat Friedmann-Robertson-Walker (FRW) metric, is described by

$$
d s^{2}=-d t \otimes d t+\sum_{i=1}^{3} a_{i}(t)^{2} d x^{i} \otimes d x^{i},
$$

where $a_{i}(t), i=1,2,3$ are the expansion factors in different spatial directions. For later convenience we define the following variables

$$
\begin{gathered}
v=\prod_{i=1}^{3} a_{i}, \\
H_{i}=\frac{\dot{a}_{i}}{a_{i}}, \quad i=1,2,3, \\
3 H=\sum_{i=1}^{3} H_{i} .
\end{gathered}
$$

In above equation, $v$ is the volume scale factor, $H_{i}, i=1,2,3$ are the directional Hubble parameters, and $H$ is the mean Hubble parameter. The physical observable are the mean anisotropy parameter $A$, the deceleration parameter $q$ and the shear scalar $\sigma^{2}$ which are defined as

$$
\begin{gathered}
A=\frac{1}{3} \sum_{i=1}^{3}\left(\frac{\Delta H_{i}}{H}\right)^{2}, \\
q=\frac{d}{d t}\left(\frac{1}{H}\right)-1=-\frac{1}{H^{2}}\left(\dot{H}+H^{2}\right), \\
\sigma^{2}=\frac{1}{2} \sigma_{i j} \sigma^{i j}=\frac{1}{2}\left(\sum_{i=1}^{3} H_{i}^{2}-3 H^{2}\right),
\end{gathered}
$$

where $\Delta H_{i}=H_{i}-H$. The sign of the deceleration parameter indicates how the universe expands. A positive sign for $q$ corresponds to a decelerating universe whereas a negative sign indicates inflation. We note that $A=0$ for an isotropic expansion.

We also assume that the confined matter source on the brane is a perfect fluid with equation of state $p=(\gamma-1) \rho$ where $1 \leq \gamma<2$.

Using the variables (27)-(29), the Einstein gravitational field equation (17), the Bianchi identity (24) and the evolution equation for non-local dark radiation (25) take the form

$$
\begin{aligned}
3 \dot{H}+\sum_{i=1}^{3} H_{i}^{2} & =\Lambda_{4}-\frac{\kappa_{4}^{2}}{2}\left[(3 \gamma-2)+\frac{\alpha_{1}}{6}(3 \gamma-4)\right] \rho-\frac{\kappa_{5}^{4}\left(\alpha_{8}+3 \alpha_{9}\right)}{2} \rho^{2}+\frac{3\left(1+\alpha_{1}\right)}{\left(3+\alpha_{1}\right)} \mathcal{U}, \\
\frac{1}{v} \frac{d}{d t}\left(v H_{i}\right) & =\Lambda_{4}-\frac{\kappa_{4}^{2}}{2}\left[(\gamma-2)+\frac{\alpha_{1}}{6}(3 \gamma-4)\right] \rho+\frac{\kappa_{5}^{4}\left(\alpha_{8}-\alpha_{9}\right)}{2} \rho^{2}-\frac{\left(1+\alpha_{1}\right)}{\left(3+\alpha_{1}\right)} \mathcal{U},
\end{aligned}
$$


and

$$
\begin{gathered}
\dot{\rho}\left[1+\alpha_{2}(4-3 \gamma)\right]+3 \gamma H \rho=0, \\
\dot{\mathcal{U}}+4 H \mathcal{U}=\frac{\kappa_{4}^{2} \gamma\left(\alpha_{2}-\frac{\alpha_{1}}{12}\right)(4-3 \gamma)\left(3+\alpha_{1}\right)}{\left(1+\alpha_{1}\right)\left[1+\alpha_{2}(4-3 \gamma)\right]} H \rho-\frac{\kappa_{5}^{4} \alpha_{10}\left(3+\alpha_{1}\right)}{\left(1+\alpha_{1}\right)\left[1+\alpha_{2}(4-3 \gamma)\right]} H \rho^{2},
\end{gathered}
$$

where $3 H=\frac{\dot{v}}{v}$ and constants $\alpha_{8}, \alpha_{9}$ and $\alpha_{10}$ are defined as

$$
\begin{gathered}
\alpha_{8}=\frac{-4\left(1+\alpha_{1}\right)\left(3+\alpha_{1}\right)+6 \gamma\left(1+\alpha_{1}\right)\left(4+\alpha_{1}\right)-3 \gamma^{2}\left(5+\alpha_{1}\right)\left(\alpha_{0}+\alpha_{1}\right)}{16\left(3+\alpha_{1}\right)\left(1+\alpha_{1}\right)\left(3-4 \alpha_{0}-\alpha_{1}\right)}, \\
\alpha_{9}=\frac{4\left(1+\alpha_{1}\right)\left(3+\alpha_{1}\right)-6 \gamma \alpha_{1}\left(1+\alpha_{1}\right)-3 \gamma^{2}\left(\alpha_{0}+\alpha_{1}\right)\left(3-\alpha_{1}\right)}{16\left(3+\alpha_{1}\right)\left(1+\alpha_{1}\right)\left(3-4 \alpha_{0}-\alpha_{1}\right)}, \\
\alpha_{10}=2 \gamma \alpha_{8}+\left(\alpha_{8}+\alpha_{9}\right)\left[1+\alpha_{2}(4-3 \gamma)\right] .
\end{gathered}
$$

For $\gamma \neq 0$ the solution of equation (35) is given by

$$
\rho=\rho_{0} v^{\frac{-\gamma}{1+(4-3 \gamma) \alpha_{2}}} .
$$

Also integrating equation (36) yields

$\mathcal{U}=-3 c v^{-4 / 3}+\frac{\kappa_{4}^{2} \rho_{0} \gamma\left(\alpha_{2}-\frac{\alpha_{1}}{12}\right)(4-3 \gamma)\left(3+\alpha_{1}\right)}{\left[4+4 \alpha_{2}(4-3 \gamma)-3 \gamma\right]\left(1+\alpha_{1}\right)} v^{\frac{-\gamma}{1+(4-3 \gamma) \alpha_{2}}}-\frac{\kappa_{5}^{4} \rho_{0}^{2} \alpha_{10}\left(3+\alpha_{1}\right)}{\left[4+4(4-3 \gamma) \alpha_{2}-6 \gamma\right]\left(1+\alpha_{1}\right)} v^{\frac{-2 \gamma}{1+(4-3 \gamma) \alpha_{2}}}$

where $c$ is a constant of integration. Using the non-local energy density and the evolution law of energy density, the gravitational field equations (33) and (34) become

$$
\begin{aligned}
3 \dot{H}+\sum_{i=1}^{3} H_{i}^{2} & =\Lambda_{4}-\frac{\kappa_{4}^{2} \rho_{0}}{2}\left[(3 \gamma-2)+\frac{\alpha_{1}}{6}(3 \gamma-4)\right] v^{\frac{-\gamma}{1+(4-3 \gamma) \alpha_{2}}}-\frac{\kappa_{5}^{4} \rho_{0}^{2}}{2}\left(\alpha_{8}+3 \alpha_{9}\right) v^{\frac{-2 \gamma}{1+(4-3 \gamma) \alpha_{2}}} \\
& +\frac{3\left(1+\alpha_{1}\right)}{\left(3+\alpha_{1}\right)} \mathcal{U}, \quad i=1,2,3 \\
\frac{1}{v} \frac{d}{d t}\left(v H_{i}\right) & =\Lambda_{4}-\frac{\kappa_{4}^{2} \rho_{0}}{2}\left[(\gamma-2)+\frac{\alpha_{1}}{6}(3 \gamma-4)\right] v^{\frac{-\gamma}{1+(4-3 \gamma) \alpha_{2}}}+\frac{\kappa_{5}^{4} \rho_{0}^{2}}{2}\left(\alpha_{8}-\alpha_{9}\right) v^{\frac{-2 \gamma}{1+(4-3 \gamma) \alpha_{2}}} \\
& -\frac{\left(1+\alpha_{1}\right)}{\left(3+\alpha_{1}\right)} \mathcal{U}, \quad i=1,2,3
\end{aligned}
$$

Summing equations (40) we find

$$
\begin{aligned}
\frac{1}{v} \frac{d}{d t}(3 v H) & =3 \Lambda_{4}-\frac{3 \kappa_{4}^{2}}{2} \rho_{0}\left[(\gamma-2)+\frac{\alpha_{1}}{6}(3 \gamma-4)\right] v^{\frac{-\gamma}{1+(4-3 \gamma) \alpha_{2}}} \\
& +\frac{3 \kappa_{5}^{4} \rho_{0}^{2}}{2}\left(\alpha_{8}-\alpha_{9}\right) v^{\frac{-2 \gamma}{1+(4-3 \gamma) \alpha_{2}}}-\frac{3\left(1+\alpha_{1}\right)}{\left(3+\alpha_{1}\right)} \mathcal{U} .
\end{aligned}
$$

Now, substituting back equation (41) into equations (40) we obtain

$$
H_{i}=H+\frac{h_{i}}{v}, \quad i=1,2,3,
$$

with $h_{i}, i=1,2,3$ being constants of integration satisfying the consistency condition $\sum_{i=1}^{3} h_{i}=0$. The basic equation describing the dynamics of the anisotropic brane-world model with Lorentz violation in the bulk can be written as

$$
\ddot{v}=3 \Lambda_{4} v+\frac{9 c\left(1+\alpha_{1}\right)}{\left(3+\alpha_{1}\right)} v^{-1 / 3}+f\left(\alpha_{0}, \alpha_{1}, \gamma\right) v^{\frac{(1-\gamma)+(4-3 \gamma) \alpha_{2}}{1+(4-3 \gamma) \alpha_{2}}}+\mathcal{F}\left(\alpha_{0}, \alpha_{1}, \gamma\right) v^{\frac{(1-2 \gamma)+(4-3 \gamma) \alpha_{2}}{1+(4-3 \gamma) \alpha_{2}}},
$$


where

$$
\begin{gathered}
f\left(\alpha_{0}, \alpha_{1}, \gamma\right)=\kappa_{5}^{4} \lambda \rho_{0} \frac{18\left(1+\alpha_{1}\right)\left[(2-\gamma)+\frac{\alpha_{1}}{6}(4-3 \gamma)\right]-\gamma\left(12 \alpha_{0}+9 \alpha_{1}+\alpha_{1}^{2}+4 \alpha_{0} \alpha_{1}\right)}{8\left(1+\alpha_{1}\right)\left(3+\alpha_{1}\right)\left(3-4 \alpha_{0}-\alpha_{1}\right)}, \\
\mathcal{F}\left(\alpha_{0}, \alpha_{1}, \gamma\right)=\kappa_{5}^{4} \rho_{0}^{2}\left[\frac{3\left[4\left(3+\alpha_{1}\right)+3 \gamma^{2}\left(\alpha_{0}+\alpha_{1}\right)-6 \gamma\left(2+\alpha_{1}\right)\right]}{16\left(3+\alpha_{1}\right)\left(3-4 \alpha_{0}-\alpha_{1}\right)}+\frac{\alpha_{10}\left(3-4 \alpha_{0}-\alpha_{1}\right)}{4\left(1+\alpha_{1}\right)+2 \gamma\left(2 \alpha_{0}-\alpha_{1}-3\right)}\right] .
\end{gathered}
$$

The general solution of equation (43) becomes

$$
\begin{aligned}
t-t_{0} & =\int\left(3 \Lambda_{4} v^{2}+\frac{27 c\left(1+\alpha_{1}\right)}{\left(3+\alpha_{1}\right)} v^{2 / 3}+2 g\left(\alpha_{0}, \alpha_{1}, \gamma\right) v^{\frac{(2-\gamma)+2(4-3 \gamma) \alpha_{2}}{1+(4-3 \gamma) \alpha_{2}}}\right. \\
& \left.+2 \mathcal{G}\left(\alpha_{0}, \alpha_{1}, \gamma\right) v^{\frac{2(1-\gamma)+2(4-3 \gamma) \alpha_{2}}{1+(4-3 \gamma) \alpha_{2}}}+C\right)^{-1 / 2} d v
\end{aligned}
$$

where

$$
\begin{gathered}
g\left(\alpha_{0}, \alpha_{1}, \gamma\right)=\frac{1+(4-3 \gamma) \alpha_{2}}{(2-\gamma)+2(4-3 \gamma) \alpha_{2}} f\left(\alpha_{0}, \alpha_{1}, \gamma\right), \\
\mathcal{G}\left(\alpha_{0}, \alpha_{1}, \gamma\right)=\frac{1+(4-3 \gamma) \alpha_{2}}{2(1-\gamma)+2(4-3 \gamma) \alpha_{2}} \mathcal{F}\left(\alpha_{0}, \alpha_{1}, \gamma\right),
\end{gathered}
$$

where $C$ is a constant of integration. The time variation of the physically important parameters described above in the exact parametric form, with $v$ taken as a parameter, is given by

$$
\begin{gathered}
A=3 h^{2}\left[3 \Lambda_{4} v^{2}+\frac{27 c\left(1+\alpha_{1}\right)}{\left(3+\alpha_{1}\right)} v^{2 / 3}+2 g\left(\alpha_{0}, \alpha_{1}, \gamma\right) v^{\frac{(2-\gamma)+2(4-3 \gamma) \alpha_{2}}{1+(4-3 \gamma) \alpha_{2}}}+2 \mathcal{G}\left(\alpha_{0}, \alpha_{1}, \gamma\right) v^{\frac{2(1-\gamma)+2(4-3 \gamma) \alpha_{2}}{1+(4-3 \gamma) \alpha_{2}}}+C\right]^{-1},(4) \\
q=2-\frac{3 v\left[3 \Lambda_{4} v+\frac{9 c\left(1+\alpha_{1}\right)}{\left(3+\alpha_{1}\right)} v^{-1 / 3}+f\left(\alpha_{0}, \alpha_{1}, \gamma\right) v^{\frac{(1-\gamma)+(4-3 \gamma) \alpha_{2}}{1+(4-3 \gamma) \alpha_{2}}}+\mathcal{F}\left(\alpha_{0}, \alpha_{1}, \gamma\right) v^{\frac{(1-2 \gamma)+(4-3 \gamma) \alpha_{2}}{1+(4-3 \gamma) \alpha_{2}}}\right]}{3 \Lambda_{4} v^{2}+\frac{27 c\left(1+\alpha_{1}\right)}{\left(3+\alpha_{1}\right)} v^{2 / 3}+2 g\left(\alpha_{0}, \alpha_{1}, \gamma\right) v^{\frac{(2-\gamma)+2(4-3 \gamma) \alpha_{2}}{1+(4-3 \gamma) \alpha_{2}}}+2 \mathcal{G}\left(\alpha_{0}, \alpha_{1}, \gamma\right) v^{\frac{2(1-\gamma)+2(4-3 \gamma) \alpha_{2}}{1+(4-3 \gamma) \alpha_{2}}}+C},(46) \\
a_{i}=a_{0 i} v^{1 / 3} \exp \left\{\int \left[3 \Lambda_{4} v^{4}+\frac{27 c\left(1+\alpha_{1}\right)}{\left(3+\alpha_{1}\right)} v^{2 / 3}+2 g\left(\alpha_{0}, \alpha_{1}, \gamma\right) v^{\frac{(2-\gamma)+2(4-3 \gamma) \alpha_{2}}{1+(4-3 \gamma) \alpha_{2}}}\right.\right. \\
+2 \mathcal{G}\left(\alpha_{0}, \alpha_{1}, \gamma\right) v^{\left.\left.\frac{2(1-\gamma)+2(4-3 \gamma) \alpha_{2}}{1+(4-3 \gamma) \alpha_{2}}+C v^{2}\right]^{-1 / 2} d v\right\}, \quad i=1,2,3} \\
\sigma^{2}=\frac{h^{2}}{2 v^{2}},
\end{gathered}
$$

where $h^{2}=\sum_{i=1}^{3} h_{i}^{2}$. Also, the integration constants $h_{i}$ and $C$ must satisfy the consistency condition $h^{2}=\frac{2}{3} C$. As one can see from equations (45)-(47) the behavior of these physical parameters depends on the Lorentz violating parameters and the equation of state of the cosmological fluid.

For a well-defined theory, the following constraints put on the parameters $\beta_{i}$ as [30]

$$
\begin{gathered}
\beta_{1} \geq 0, \\
\left(\beta_{1}+\beta_{2}+\beta_{3}\right) / \beta_{1} \leq 1, \\
\left(\beta_{1}+\beta_{2}+\beta_{3}\right) / \beta_{1} \geq 0, \\
\beta_{1}+\beta_{3} \leq 0 .
\end{gathered}
$$




\begin{tabular}{|c|c|c|c|c|}
\hline \multicolumn{2}{|c|}{ constraints for $G>0$} & $\bar{G}$ & constraints of ref [30] & integer values of $\alpha_{0}$ and $\alpha_{1}$ \\
\hline \multirow[t]{2}{*}{ 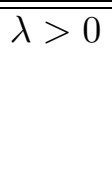 } & $\begin{array}{c}3+\alpha_{1}>0 \\
3-4 \alpha_{0}-\alpha_{1}>0\end{array}$ & positive & $\begin{array}{c}\alpha_{0} \geq 0 \\
\alpha_{0}+\alpha_{1} \geq 0\end{array}$ & $\alpha_{0}=0, \alpha_{1}=0,1,2$ \\
\hline & $\begin{array}{c}3+\alpha_{1}<0 \\
3-4 \alpha_{0}-\alpha_{1}<0\end{array}$ & positive & $\begin{array}{c}\alpha_{0} \geq 0 \\
\alpha_{0}+\alpha_{1} \geq 0\end{array}$ & $\begin{array}{c}\alpha_{0}=\{4,5,6, \ldots\} \\
\alpha_{1}=\left\{-\alpha_{0},-\alpha_{0}+1,-\alpha_{0}+2 \ldots,-4\right\}\end{array}$ \\
\hline \multirow[t]{2}{*}{$\lambda<0$} & $\begin{array}{c}3+\alpha_{1}<0 \\
3-4 \alpha_{0}-\alpha_{1}>0\end{array}$ & positive & $\begin{array}{c}\alpha_{0} \geq 0 \\
\alpha_{0}+\alpha_{1} \geq 0\end{array}$ & no values \\
\hline & $\begin{array}{c}3+\alpha_{1}>0 \\
3-4 \alpha_{0}-\alpha_{1}<0\end{array}$ & positive & $\begin{array}{c}\alpha_{0} \geq 0 \\
\alpha_{0}+\alpha_{1} \geq 0\end{array}$ & $\begin{array}{c}\alpha_{0}=0, \alpha_{1}>3 \\
\alpha_{0}=1, \alpha_{1}>-1 \\
\alpha_{0}=\{2,3,4, \ldots\}, \alpha_{1}>-3\end{array}$ \\
\hline
\end{tabular}

Table 1: The possible values of Lorentz violating parameters in a brane-world universe.

The first condition results from the need for a positive-definite Hamiltonian for the perturbations; the next two from demanding the subluminal and non-tachyonic propagation of the spin-0 field; and the last condition from insisting subluminal propagation of the spin-2 field. Together these conditions imply that

$$
\begin{gathered}
\beta_{1}+\beta_{2}+\beta_{3} \geq 0, \\
\beta_{2} \geq 0,
\end{gathered}
$$

which in terms of $\alpha_{0}$ and $\alpha_{1}$ we have

$$
\begin{gathered}
\alpha_{0}+\alpha_{1} \geq 0, \\
\alpha_{0} \geq 0 .
\end{gathered}
$$

The above conditions together with constraints require for a positive effective Newtonian constant (19), lead to the particular values of $\alpha_{0}$ and $\alpha_{1}$. We have summarized these results in table 1 .

The singular state at $t=0$ is characterized by the condition $v(0)=0$. The value of the anisotropy parameter for $t=0$ depends on the Lorentz violating parameters and the equation of the state. Hence for $\gamma=1$ equation (45) reduces to

$$
A=\frac{3 h^{2}}{3 \Lambda_{4} v^{2}+\frac{27 c\left(1+\alpha_{1}\right)}{\left(3+\alpha_{1}\right)} v^{\frac{2}{3}}+2 g\left(\alpha_{0}, \alpha_{1}, 1\right) v^{\frac{1+2 \alpha_{2}}{1+\alpha_{2}}}+2 \mathcal{G}\left(\alpha_{0}, \alpha_{1}, 1\right) v^{\frac{2 \alpha_{2}}{1+\alpha_{2}}}+C},
$$

the singular behavior of the dust filled brane universe depends on $\alpha_{2}$, so that from above equation it follows

$$
\lim _{v \rightarrow 0} A(v)=0, \quad-1<\alpha_{2}<0,
$$

and

$$
\lim _{v \rightarrow 0} A(v)=\frac{36 h^{2}}{k_{5}^{4} \rho_{0}^{2}+12 C}, \quad \alpha_{2}=0
$$

and otherwise

$$
\lim _{v \rightarrow 0} A(v)=\frac{3 h^{2}}{C} .
$$

In the absence of the Lorentz violating parameters the dust filled brane universes are born in an anisotropic state [32], while in the brane universe with the Lorentz invariance violation the early time behavior of the brane universe is sensitive to the values of $\alpha_{2}$, so that it is possible to admitting of both the isotropic and anisotropic state. Therefore the singular state of the dust filled Bianchi type I brane universe in our model is different from the case of brane cosmological models without the bulk Lorentz violation. 
For the case of the radiation anisotropic brane universe, $p=\frac{1}{3} \rho$ and $\gamma=\frac{4}{3}$, equation (45) reduces to

$$
A=\frac{3 h^{2}}{3 \Lambda_{4} v^{2}+\frac{27 c\left(1+\alpha_{1}\right)}{\left(3+\alpha_{1}\right)} v^{\frac{2}{3}}+2 g\left(\alpha_{0}, \alpha_{1}, 4 / 3\right) v^{\frac{2}{3}}+2 \mathcal{G}\left(\alpha_{0}, \alpha_{1}, 4 / 3\right) v^{-\frac{2}{3}}+C},
$$

in this case the singular behavior of the anisotropy parameter is independent of $\alpha_{2}$, so that from equation (53), we have

$$
\lim _{v \rightarrow 0} A(v)=0 .
$$

In figure 1, we have plotted the anisotropy parameter for $\alpha_{0}=0$ and $\alpha_{1}=2$. Since $\alpha_{2}$ expressed in terms of $\alpha_{0}$ and $\alpha_{1}$ becomes $\alpha_{2}=\frac{\alpha_{0}+\alpha_{1}}{3-4 \alpha_{0}-\alpha_{1}}$, therefore $\alpha_{2}=2$ and from equation (52) for $\gamma=1$ we have $A(0)=\frac{3 h^{2}}{C}$ and the anisotropic brane universe starts its evolution from an anisotropic state to an isotropic de Sitter inflationary phase at late time. For $\gamma=4 / 3$, the late time behavior of anisotropy parameter is isotropic with $A(0)=0$. The behavior of the deceleration parameter of the Bianchi type I geometry is illustrated, for different values of $\gamma, \alpha_{0}=0$ and $\alpha_{1}=2$, in figure 2 . In the initial stage the behavior of the Bianchi type I brane universe is non-inflationary, but brane universe ends in an accelerated expanding stage at late time. The time variation of the shear parameter as a function of time is represented in figure 3. The shear is a decreasing function of time and in the limit of large time corresponding to the isotropic limit, $\sigma^{2} \rightarrow 0$. In the limit of small time the shear has a singular behavior tending to infinity, $\sigma^{2} \rightarrow \infty$.

For $\beta_{2}=0$ and $\beta_{1}=-\beta_{3}$, equations (45)-(47) give the general solutions of the physical parameters on the volume scale factor for the generalized RS II model [33]

$$
\begin{gathered}
a_{i}=a_{0 i} v^{1 / 3} \exp \left[h_{i} \int\left(3 \Lambda_{4} v^{4}+3 \kappa_{4}^{2} \rho_{0} v^{4-\gamma}+\frac{1}{4} \kappa_{5}^{4} \rho_{0}^{2} v^{4-2 \gamma}+C v^{2}\right)^{-1 / 2} d v\right], \quad i=1,2,3, \\
q=2-\frac{36 \Lambda_{4} v^{2}+18(2-\gamma) \kappa_{4}^{2} \rho_{0} v^{2-\gamma}+3(1-\gamma) \kappa_{5}^{4} \rho_{0}^{2} v^{2-2 \gamma}}{12 \Lambda_{4} v^{2}+12 \kappa_{4}^{2} \rho_{0} v^{2-\gamma}+\kappa_{5}^{4} \rho_{0}^{2} v^{2-2 \gamma}+4 C} \\
A=3 h^{2}\left(3 \Lambda_{4} v^{2}+3 \kappa_{4}^{2} \rho_{0} v^{2-\gamma}+\frac{1}{4} \kappa_{5}^{4} \rho_{0}^{2} v^{2-2 \gamma}+C\right)^{-1} \\
\sigma^{2}=\frac{h^{2}}{2 v^{2}}
\end{gathered}
$$

where $h^{2}=\sum_{i=1}^{3} h_{i}^{2}$ and $C$ is a constant of integration. The behavior of the deceleration parameter of the Bianchi type I geometry is illustrated, for different values of $\gamma$, in figure 4 . In the initial stage the behavior of the Bianchi type I brane universe is non-inflationary, but brane universe ends in an accelerated expanding stage at late time.

In figure 5, we have also plotted the anisotropy parameter for different values of $\gamma$. The behavior of the anisotropy parameter shows that at high densities the brane universe starts its evolutions from an isotropic state with $A(0)=0$ for $\gamma=4 / 3$, and ends in an isotropic de Sitter inflationary phase at late time. An important difference between the anisotropic homogeneous brane-world cosmological models and the standard general relativity is that brane universes are born in an isotropic state.

The time variation of the shear parameter as a function of time is represented in figure 6 . The shear is a decreasing function of time and in the limit of large time corresponding to the isotropic limit, $\sigma^{2} \rightarrow 0$. In the limit of small time the shear has a singular behavior tending to infinity, $\sigma^{2} \rightarrow \infty$. 


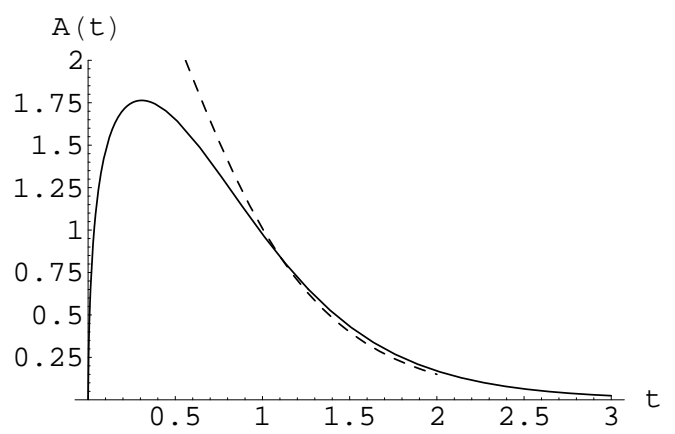

Figure 1: The anisotropy parameter of the Bianchi type I brane geometry, with the bulk Lorentz violation $\alpha_{0}=0$, $\alpha_{1}=2$, for $\gamma=4 / 3$ (solid curve) and for $\gamma=1$ (dashed curve).

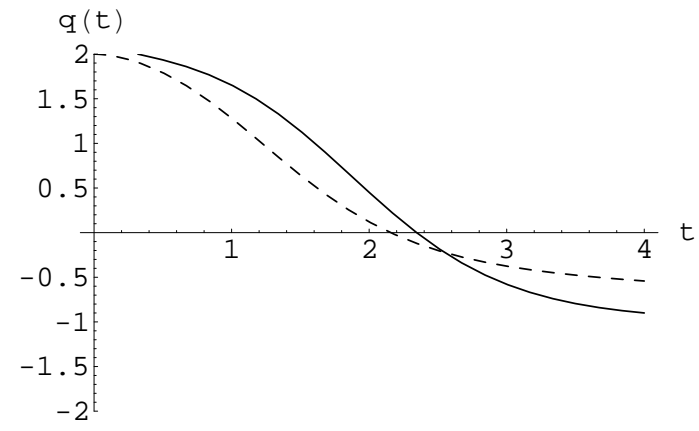

Figure 2: The deceleration parameter of the Bianchi type I brane geometry, with the bulk Lorentz violation $\alpha_{0}=0$, $\alpha_{1}=2$, for $\gamma=4 / 3$ (solid curve) and for $\gamma=1$ (dashed curve).

\section{Conclusions}

In this letter, we have dealt with the dark energy problem in the context of the brane-world scenario with the bulk Lorentz violation, introduced by specifying a preferred frame through the introduction of a dynamical vector field normal to the brane. In our model, due to the local Lorentz violation in the bulk, the Friedmann equations on the anisotropic brane have been modified by the Lorentz violating parameters $\beta_{i}, i=1,2,3$ and the equation of state of the matter. Therefore, for a fixed value of $\gamma$, the behavior of an anisotropic brane universe is controlled by the Lorentz violating parameters. The behavior of the anisotropy parameter shows that the brane universe evolves from an isotropic/anisotropic state to an isotropic state which has entered an accelerated expanding phase. The early time behavior of anisotropic parameter is different from those obtained in RS type II brane-world models without the bulk Lorentz violation [33].

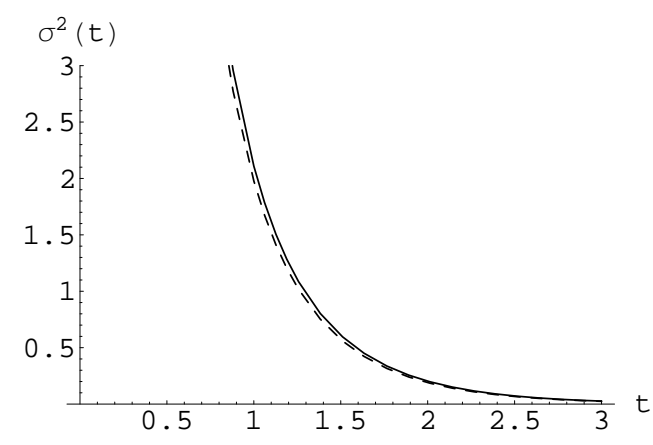

Figure 3: The shear parameter of the Bianchi type I brane universe, with the bulk Lorentz violation $\alpha_{0}=0, \alpha_{1}=2$, for $\gamma=4 / 3$ (solid curve) and for $\gamma=1$ (dashed curve). 


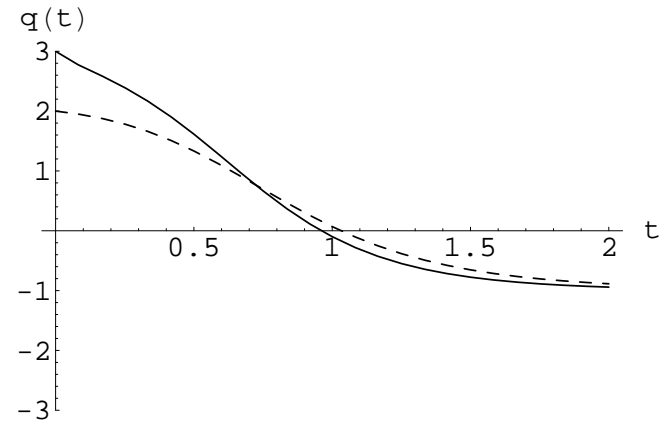

Figure 4: The deceleration parameter of the Bianchi type I brane universe with confined perfect cosmological fluid for $\gamma=4 / 3$ (solid curve) and $\gamma=1$ (dashed curve).

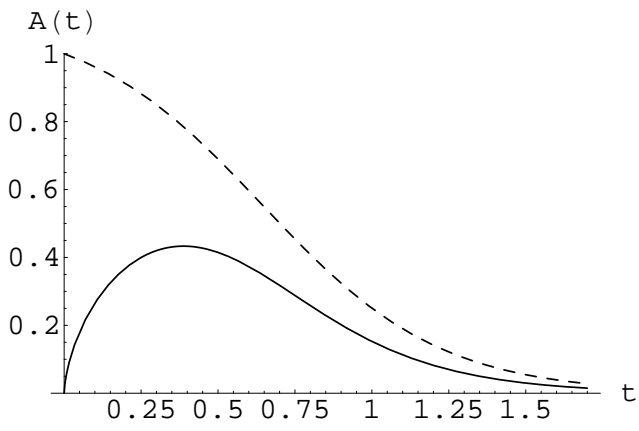

Figure 5: The anisotropy parameter of the Bianchi type I brane universe with confined perfect cosmological fluid for $\gamma=4 / 3$ (solid curve) and $\gamma=1$ (dashed curve).

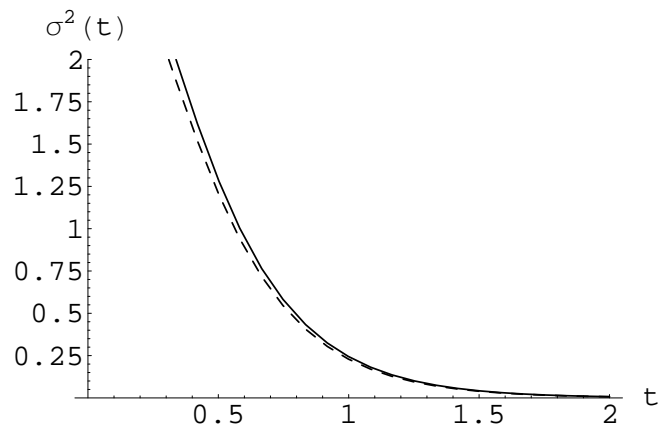

Figure 6: The shear parameter of the Bianchi type I brane universe with confined perfect cosmological fluid for $\gamma=4 / 3$ (solid curve) and $\gamma=1$ (dashed curve). 


\section{References}

[1] L. Randall and R. Sundrum, Phys. Rev. Lett. 833370 (1999); ibid 834690 (1999).

[2] G. Dvali, G. Gabadadze and M. Porrati, Phys. Lett. B 485208 (2000);

G. Dvali and G. Gabadadze, Phys. Rev. D 63065007 (2001).

[3] P. Kraus, JHEP 9912011 (1999);

H. Collins and B. Holdom, Phys. Rev. D 62105009 (2000).

[4] W. Israel, Nouvo Cimento B 441 (1966).

[5] T. Shiromizu, K. Maeda and M. Sasaki, Phys. Rev. D 62024012 (2000).

[6] P. Brax and C. van de Bruck, Class. Quant. Grav. 20 R201 (2003);

P. Brax, C. van de Bruck and A. C. Davis, Rep. Prog. Phys. 672183 (2004).

[7] P. Binetruy, C. Deffayet and D. Langlois, Nucl. Phys. B 565269 (2000);

P. Binetruy, C. Deffayet, U. Ellwanger and D. Langlois, Phys. Lett. B 477285 (2000).

[8] R. Maartens, Living Rev. Rel. 77 (2004).

[9] M. S. Berger and V. A. Kostelecky, Phys. Rev. D 65091701 (2002).

[10] A. Connes, M. R. Douglas and A. Schwarz, JHEP 02003 (1998).

[11] S. M. Carroll, J. A. Harvey, V. A. Kostelecky, C. D. Lane and T. Okamoto, Phys. Rev. Lett. 87 141601 (2001).

[12] G. T. Zatsepin and V. A. Kuzmin, Prima Zh. Eksp. Teor. Fiz 4114 (1966).

[13] S. L. Dubovsky and P. G. Tinyakov, Astropart. Phys. 1889 (2002);

J. R. Chisholm and E. W. Kolb, Phys. Rev. D 69085001 (2004);

S. R. Coleman and S. L. Glashow, Phys. Rev. D 59116008 (1999).

[14] H. Vankov and T. Stanev, Phys. Lett. B 538251 (2002);

T. Kifune, Astrophys. J. 518 L21 (1999);

A. Zee, Phys. Rev. D 251864 (1982).

[15] R. Aloisio, P. Blasi, P. L. Ghia, and A. F. Grillo, Phys. Rev. D 62053010 (2000);

O. Bertolami and C. S. Carvalho, Phys. Rev. D 61103002 (2000);

S. R. Coleman and S. L. Glashow, Phys. Lett. B 405249 (1997).

[16] J. Alfaro and G. Palma, Phys. Rev. D 67083003 (2003);

O. Bertolami, Nucl. Phys. Proc. Suppl. 8849 (2000);

O. Gagnon and G. D. Moore, Phys. Rev. D 70065002 (2004).

[17] V. A. Kostelecky and S. Samuel, Phys. Rev. D 401886 (1989).

[18] J. D. Bekenstein, Phys. Rev. D 70083509 (2004).

[19] K. J. Nordtvedt and C. M. Will, Astrophys. J. 177775 (1972);

C. Armendariz-Picon, JCAP 0407007 (2004);

L. H. Ford, Phys. Rev. D 40967 (1989).

[20] A. D. Dolgov, JETP Lett. 41345 (1985);

V. V. Kiselev, Class. Quant. Grav. 22541 (2005);

V. V. Kiselev, Class. Quant. Grav. 213323 (2004). 
[21] D. Colladay and V. A. Kostelecky, Phys. Rev. D 58116002 (1998);

S. M. Carroll, G. B. Field, and R. Jackiw, Phys. Rev. D 411231 (1990).

[22] K. Nozari and S. D. Sadatian, JCAP 0901005 (2009);

K. Farakos, JHEP 0908031 (2009);

C. Csaki, J. Erlich and C. Grojean, Gen. Rel. Grav. 331921 (2001);

M. V. Libanov and V. A. Rubakov, Phys. Rev. D 72123503 (2005);

O. Bertolami and C. Carvalho, Phys. Rev. D 74084020 (2006);

T. G. Rizzo, JHEP 1011156 (2010) ;

J. Soda and S. Kanno, (2006) [arXiv:gr-qc/0612069].

[23] C. Csaki, J. Erlich and C. Grojean, Nucl. Phys. B 604312 (2001);

C. P. Burgess, J. Cline, E. Filotas, J. Matias and G. D. Moore, JHEP 0203043 (2002).

[24] H. Stoica, JHEP 0207060 (2002).

[25] M. V. Libanov and V. A. Rubakov, JCAP 0509005 (2005).

[26] F. Ahmadi, S. Jalalzadeh and H. R. Sepangi, Class. Quant. Grav. 234069 (2006).

[27] F. Ahmadi, S. Jalalzadeh and H. R. Sepangi, Phys. Lett. B 647486 (2007).

[28] T. Jacobson and D. Mattingly, Phys. Rev. D 64024028 (2001).

[29] V. A. Kostelecky, Phys. Rev. D 69105009 (2004);

F. Can, D. Bear and et. al., Phys. Rev. Lett. 93230801 (2004).

[30] S. M. Carroll and E. A. Lim, Phys. Rev. D 70123525 (2004);

E. A. Lim, Phys. Rev. D71 063504 (2005).

[31] M. Heydari-Fard and H. R. Sepangi, Phys. Rev. D 76104009 (2007).

[32] T. Harko and M. K. Mak, Class. Quant. Grav. 211489 (2004).

[33] C. M. Chen, T. Harko and M. K. Mak, Phys. Rev. D 64044013 (2001). 